Z Epileptol 2018 $\cdot 31: 231-232$ https://doi.org/10.1007/s10309-018-0211-0 Online publiziert: 25 . September 2018 (c) Springer Medizin Verlag $\mathrm{GmbH}$, ein Teil von Springer Nature 2018

CrossMark

\author{
Adam Strzelczyk ${ }^{1}$ Christoph Kellinghaus ${ }^{2} \cdot$ Eugen Trinka $^{3}$ \\ 'Epilepsiezentrum Frankfurt Rhein-Main, Zentrum der Neurologie und Neurochirurgie, \\ Universitätsklinikum Frankfurt, Frankfurt am Main, Deutschland \\ ${ }^{2}$ Klinik für Neurologie, Epilepsiezentrum Münster-Osnabrück, Klinikum Osnabrück, Osnabrück, \\ Deutschland \\ ${ }^{3}$ Universitätsklinik für Neurologie, Christian-Doppler-Klinik, Paracelsus Medizinische Universität Salzburg, \\ Salzburg, Österreich
}

\section{Update zum Status epilepticus}

Der Status epilepticus (SE) gehört zu den wichtigsten neurologischen und zunehmend häufiger diagnostizierten Notfällen und bleibt wissenschaftlich ein hochaktuelles Thema sowohl in den klinischen wie grundlagenorientierten Neurowissenschaften. Bereits im Vorwort zum Themenheft 02/2013 zum Status epilepticus [1] wurde auf eine außerordentliche Zunahme neuer Daten hingewiesen: In PubMed fanden die Autoren für die Jahre 1960 bis 1975 weniger als 300 Publikationen, von 2006 bis 2013 über 3500, mittlerweile kommen jährlich mehr als 600 neue Publikationen dazu (- Abb. 1).

Um diese Daten für den klinischen Alltag aufzubereiten, scheint es mehr als berechtigt, ein erneutes Themenheft der Zeitschrift für Epileptologie zum Status epilepticus zu konzipieren. Zwischen- zeitlich sind in 2015 neue Diagnoseund Klassifikationskriterien des SE veröffentlicht worden [2], zudem liegen validierte Kriterien für die Diagnose eines nonkonvulsiven SE vor [3, 4].

Schwerpunkte dieses Heftes sind die Diagnosekriterien [5, 6], ein Update zur Epidemiologie [4] sowie Prognoseindikatoren und neuere Versorgungsdaten. Vier Artikel widmen sich der stufenadaptierten Therapie des SE. Besonderheiten zur Behandlung des SE bei Kindern und Jugendlichen werden von S. SchubertBast im Themenheft 2/2019 zu Pädiatrischer Epileptologie behandelt.

Mehrere Studien geben Hinweise auf eine inadäquate Therapie von prolongierten Anfällen, Anfallsclustern oder des SE im häuslichen Umfeld und im Rettungsdienst. In der Prähospitalphase gibt es deutliche Schwierigkeiten, einen SE zu
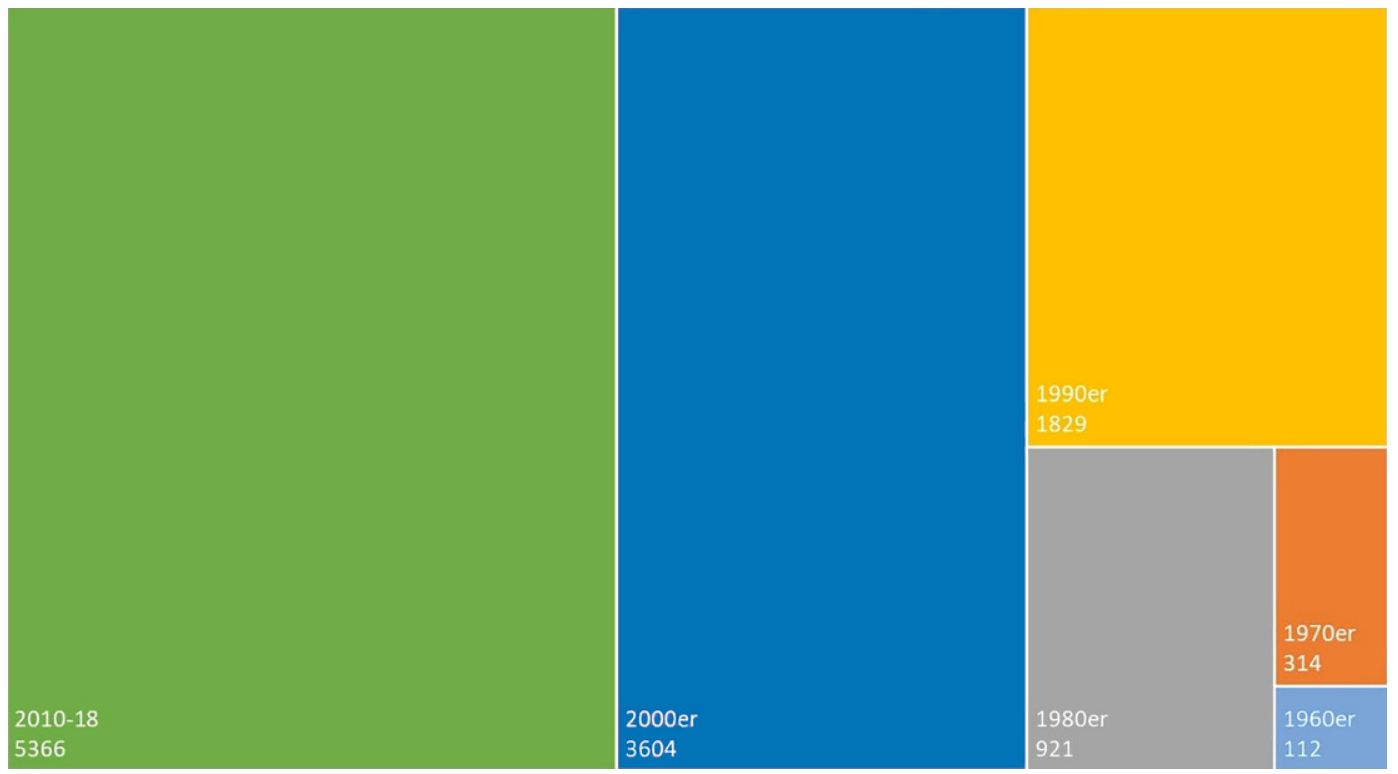

Abb. $1<$ Treemap der in PubMed gelisteten Publikationen zum Status epilepticus, gruppiert nach Erscheinungsjahrzehnt ab 1960 (Search term: Status epilepticus; Stand 12.09.2018) 
erkennen [7, 8]. In der Übersicht von G. Möddel et al. wird neben den Diagnosekriterien ausführlich die initiale Therapie des SE besprochen, insbesondere unter Berücksichtigung neuer Applikationsformen von Benzodiazepinen, die sicherlich bald auch Eingang in die Leitlinien finden werden [9].

Der Frage, welches Antikonvulsivum als Erstes nach den Benzodiazepinen eingesetzt werden soll, geht der Artikel von G. Kalss et al. nach, in dem Phenytoin, Valproat, Levetiracetam und Lacosamid verglichen werden [10]. Kohortenstudien zeigen deutlich, dass im SE trotz divergierender Empfehlung der Leitlinien v. a. Levetiracetam als Mittel der ersten Wahl vor Valproat und Lacosamid eingesetzt wird. Mittlerweile scheint Phenytoin in der klinischen Praxis als Reservemittel eingesetzt $\mathrm{zu}$ werden [11-13]. Welche teilweise nur oral verfügbaren Antikonvulsiva danach infrage kommen, wird von Rohracher et al. unter besonderer Berücksichtigung von Brivaracetam, Perampanel und Topiramat erörtert [14]. Da eine Eskalation der Therapie zur Intubationsnarkose auch Risiken birgt, die in mehreren Studien mit einer Erhöhung der Morbidität und Mortalität beschrieben wurden $[15,16]$, ist diesem Thema ein eigener Beitrag von V. Alvarez und A. Rossetti gewidmet [17].

Abschließend fassen L. Willems und L. Kay mit Kollegen den mit dem SE assoziierten Ressourcenverbrauch und die Mortalität zusammen [18]. In den letzten 5 Jahren sind hierzu mehrere Studien publiziert worden, die die Versorgungsaspekte und die Bedeutung des SE innerhalb der mit epileptischen Anfällen und Epilepsien verbundenen Erkrankungen deutlich aufzeigen $[19,20]$.

Wir hoffen, dass diese Übersichten Ihr Interesse finden werden.

\section{Ihre}

Prof. Dr. Adam Strzelczyk

Priv.-Doz. Dr. Christoph Kellinghaus

Prof. Dr. Eugen Trinka

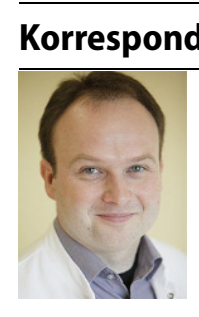

7. Kadel J, Bauer S, Hermsen AM, Immisch I, Kay L, Klein KM et al (2018) Use of emergency medication in adult patients with epilepsy: a multicentre cohort study from Germany. CNS Drugs 32(8):771-781

8. Semmlack S, Yeginsoy D, Spiegel R, Tisljar K, Ruegg S, Marsch S et al (2017) Emergency response to out-of-hospital status epilepticus: A 10-year observational cohort study. Neurology 89(4):376-384

9. Möddel G, Kellinghaus C, Strzelczyk A (2018) Initiale Therapie des Status epilepticus: Welche Therapie erfolgt zu Hause und im Rettungswagen? Z Epileptol. https://doi.org/10.1007/s10309-0180204-z

10. Kalss G etal (2018) Phenytoin, Valproat, Levetiracetam und Lacosamid im Status epilepticus: Welches Mittel ist erste Wahl und wann einzusetzen? Z Epileptol. https://doi.org/10.1007/s10309-0180207-9

11. Kellinghaus C, Stogbauer F (2012) Treatment of status epilepticus in a large community hospital. Epilepsy Behav 23(3):235-240

12. Kortland LM, Alfter A, Bahr O, Carl B, Dodel R, Freiman TM et al (2016) Costs and cost-driving factors for acute treatment of adults with status epilepticus: a multicenter cohort study from Germany. Epilepsia 57(12):2056-2066

13. Kellinghaus C, Rossetti AO, Trinka E, Lang N, Unterberger l, Ruegg S et al (2018) SENSE registry for status epilepticus. Epilepsia. https://doi.org/10 1111/epi.14495

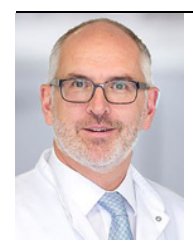
christoph.kellinghaus@ klinikum-os.de

\section{Univ. Prof. Dr. Mag.} Eugen Trinka, FRCP Universitätsklinik für Neurologie, ChristianDoppler-Klinik, Paracelsus Medizinische Universität Salzburg 5020 Salzburg, Österreich e.trinka@salk.at

Interessenkonflikt. A. Strzelczyk, C. Kellinghaus und E. Trinka geben an, dass bezüglich des Editorials kein Interessenkonflikt besteht.

\section{Literatur}

1. Rosenow F, Trinka E (2013) Status epilepticus Integraler Bestandteil epileptologischer und neurowissenschaftlicher Forschung. Z Epileptol 26(2):69

2. Trinka $E$, Cock $H$, Hesdorffer D, Rossetti AO, Scheffer IE, Shinnar S et al (2015) A definition and classification of status epilepticus - report of the ILAE Task Force on Classification of Status Epilepticus. Epilepsia 56(10):1515-1523

3. Leitinger M, Trinka E, Gardella E, Rohracher A, Kalss G, Qerama E et al (2016) Diagnostic accuracy of the Salzburg EEG criteria for non-convulsive status epilepticus: a retrospective study. Lancet Neurol 15(10):1054-1062

4. Knake S, Leitinger M (2018) Epidemiologie des Status epilepticus. Z Epileptol. https://doi.org/10. 1007/s10309-018-0213-y

5. Trinka E, Leitinger M (2018) Neue Definition und Klassifikation des Status epilepticus - Was ändert sich für die Praxis? Z Epileptol. https://doi.org/10. 1007/s10309-018-0214-X

6. Leitinger M, Trinka E (2018) Non-konvulsiver Status epilepticus. Z Epileptol. https://doi.org/10.1007/ s10309-018-0212-z
14. Rohracher A, Kellinghaus C, Strzelczyk A (2018) Topiramat, Perampanel und Brivaracetam im Status epilepticus: Wie anzuwenden und was können wir erwarten? Z Epileptol. https://doi.org/ 10.1007/s10309-018-0206-x

15. Sutter R, Marsch S, Fuhr P, Kaplan PW, Ruegg $S$ (2014) Anesthetic drugs in status epilepticus: risk or rescue? A 6-year cohort study. Neurology 82(8):656-664

16. Marchi NA, Novy J, Faouzi M, Stahli C, Burnand B, Rossetti AO (2015) Status epilepticus: impact of therapeutic coma on outcome. Crit Care Med 43(5):1003-1009

17. Alvarez V, Rossetti AO (2018) Therapeutic coma for the treatment of status epilepticus. Z Epileptol. https://doi.org/10.1007/s10309-018-0194-X

18. Willems LM, Kay L, Rosenow F, Strzelczyk A (2018) Krankheitskosten und Outcome: Versorgungsrealität des Status epilepticus. Z Epileptol. https://doi. org/10.1007/s10309-018-0205-y

19. Kortland LM, Knake S, von Podewils F, Rosenow F, Strzelczyk A (2017) Socioeconomic outcome and quality of life in adults after status epilepticus: a multicenter, longitudinal, matched case-control analysis from Germany. Front Neurol 8:507

20. StrzelczykA, AnsorgeS, Hapfelmeier J, Bonthapally V, Erder MH, Rosenow F (2017) Costs, length of stay, and mortality of super-refractory status epilepticus: a population-based study from Germany. Epilepsia 58(9):1533-1541 